\title{
UMA ANÁLISE DE FOTOGRAFIAS DE VÍTIMAS DE ABUSO SEXUAL DO PROJETO UNBREAKABLE USANDO A GRAMÁTICA DO DESIGN VISUAL
}

\author{
Victor Gomes Milani', Odair Benedito Franciso² \\ 'Universidade Federal de Santa Maria, Curso de Mestrado em Letras, Santa Maria, RS. ${ }^{2}$ Universidade do Oeste \\ Paulista - UNOESTE, tutor em cursos de pós-graduação lato sensu EaD, Presidente Prudente, SP. Universidade Estadual \\ Paulista - UNESP, Mestrado em Educação, Presidente Prudente, SP. E-mail: lordemilani@yahoo.com.br. \\ CAPES - Comissão de Aperfeiçoamento de Pessoal de Nível Superior
}

\section{RESUMO}

Os gêneros multimodais se tornam cada vez mais interesse de estudos de linguistas aplicados (BEZERRA; NASCIMENTO, 2013). O objetivo desse estudo foi analisar fotografias de vítimas de abuso sexual utilizando a Gramática do Design Visual (KRESS, VAN LEEUWEN; 2006) como ferramenta analítica. As fotografias aqui analisadas foram submetidas à publicação pelas próprias vítimas através do projeto unbreakable na rede social Tumblr. Foram selecionadas vinte fotografias publicadas anteriormente a primeiro de maio de 2016. Os resultados apontaram uma preferência para estruturas conceituais, demandas e configurações topo/base. Concluiu-se que essas escolhas se deram em virtude do propósito do projeto: dar voz às vítimas de abuso sexual (PROJETO UNBREAKABLE; 2016).

Palavras-chave: Multimodalidade; Gramática do Design Visual; Projeto Unbreakable.

\section{AN ANALYSIS OF SEXUAL ASSAULT VICTIMS' PHOTOGRAPHS OF THE UNBREAKABLE PROJECT USING THE GRAMMAR OF VISUAL DESIGN}

\begin{abstract}
Multimodal genres have increasingly become interesting for applied linguists (BEZERRA; NASCIMENTO, 2013). This study aimed at analyzing sexual assault victims' photographs of the unbreakable project using the Grammar of Visual Design (KRESS, VAN LEEUWEN; 2006) as analytical tool. The photographs analyzed here were submitted for publication by the victims themselves through the unbreakable project via the social network Tumblr. Twenty photographs published before May $1^{\text {st }} 2016$ were selected. Results showed a preference for conceptual structures, demands and topo/bottom configurations. We concluded that these choices were due to the purpose of the project: to give voice to victims of sexual assault (PROJETO UNBREAKABLE; 2016).
\end{abstract}

Keywords: Multimodality; Grammar of Visual Design; Unbreakable Project. 


\section{INTRODUÇÃO}

A produção de gêneros textuais que utilizam mais de um modo semiótico em sua composição (palavra escrita, imagem, som, etc.) cresce constantemente. Por isso, esses textos, que podem ser chamados de multimodais são encontrados em inúmeras esferas de conhecimento, desde o quotidiano até a academia e acabam se tornando objeto de pesquisa de linguistas aplicados (BEZERRA; NASCIMENTO, 2013). Uma ferramenta analítica para o estudo de imagens, por exemplo, pode ser percebida na Gramática do Design Visual (GVD) (KRESS, VAN LEEUWEN; 2006), essa gramática propõe categorizações para as imagens estáticas em termos de significados representacionais, interativos e composicionais, que são inspirados nas metafunções ideacional, interpessoal e textual da Gramática Sistêmico-Funcional (HALLIDAY, 2004; 2014) respectivamente. A GVD se constitui como uma ferramenta analítica para linguistas aplicados melhor compreenderem o papel das imagens estáticas nos gêneros multimodais a fim de contribuir para o desenvolvimento dos Multiletramentos (COPE, KALANTZIS; 2000) tanto na educação básica quanto no ensino superior.

O objetivo desse estudo foi analisar fotografias de vítimas de abuso sexual utilizando a Gramática do Design Visual (KRESS, VAN LEEUWEN; 2006) como ferramenta analítica. As fotografias aqui analisadas foram submetidas à publicação pelas próprias vítimas através do projeto unbreakable na rede social Tumblr. O projeto unbreakable teve como objetivo dar voz a sobreviventes de abuso sexual, violência doméstica e abuso infantil através da publicação de fotografias das vítimas com algum dizer dos abusadores ou de pessoas que souberam do abuso (PROJETO UNBREAKABLE; 2016). Na próxima seção, explicaremos como se deram a seleção do corpus e os passos de análise.

\section{METODOLOGIA}

\section{Seleção do corpus e Procedimentos Analíticos}

As fotografias analisadas seguiram apenas um critério de seleção: elas deveriam apresentar algum elemento que lhes desse o caráter de um gênero multimodal, ou seja, outros elementos em sua composição além dos verbais. Muitas fotografias publicadas pelo projeto unbreakable apresentaram apenas elementos verbais uma vez que as vítimas preferiram não ser identificadas. Para um melhor entendimento desse critério, apresentamos a seguir exemplos de fotografias que foram e não foram selecionadas para a análise:

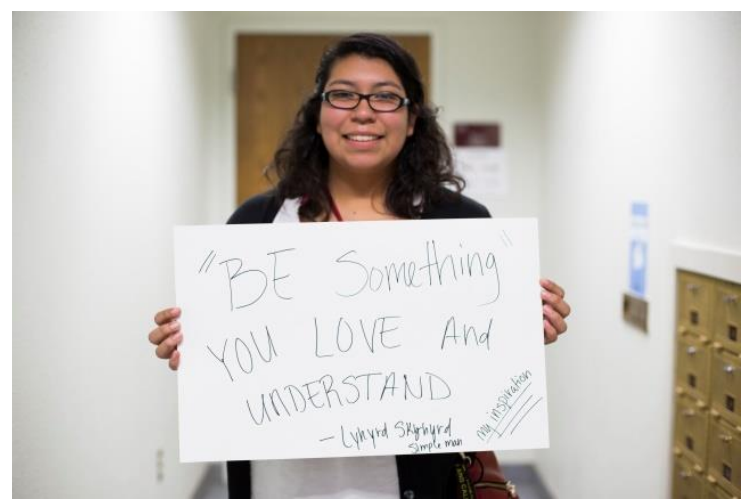

Figura 1. Exemplo de fotografia com elementos verbais (dizer) e não verbais (participante humano, cenário), segue o critério de seleção. (F\#1) 


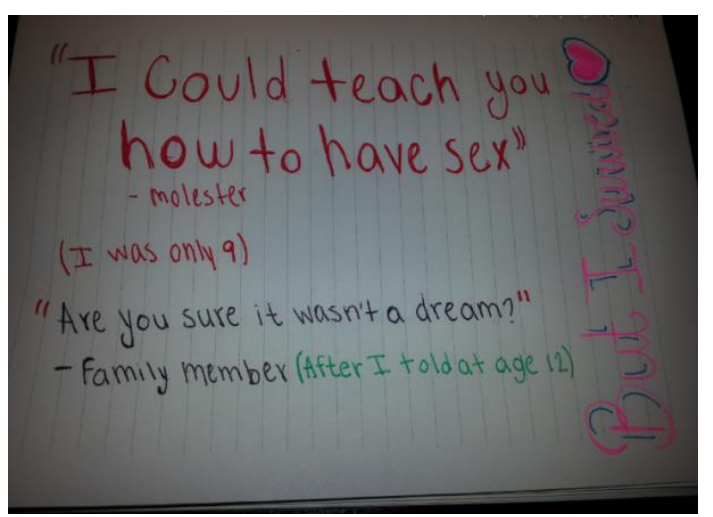

Figura 2. Exemplo de fotografia com elementos verbais apenas (dizer) não segue o critério de seleção.

Centenas de fotografias encaixaram-se no critério de seleção. Não sendo possível a análise de todas no presente estudo, optamos pela coleta das últimas 20 fotografias publicadas pelo projeto a partir data de 1 o de maio de 2016. A Tabela 1 traz a data de publicação das 20 fotografias que compõe o corpus do estudo:

Tabela 1. Datas de publicação do corpus do estudo.

\begin{tabular}{|c|c|}
\hline Número da fotografia & Data de Publicação \\
\hline F\#1 & 14 de abril de 2015 \\
\hline F\#2 & 14 de abril de 2015 \\
\hline F\#3 & 14 de abril de 2015 \\
\hline F\#4 & 14 de abril de 2015 \\
\hline F\#5 & 14 de abril de 2015 \\
\hline F\#6 & 14 de abril de 2015 \\
\hline F\#7 & 15 de março de 2015 \\
\hline F\#8 & 15 de março de 2015 \\
\hline F\#9 & 15 de março de 2015 \\
\hline F\#10 & 15 de março de 2015 \\
\hline F\#11 & 15 de março de 2015 \\
\hline F\#12 & 15 de março de 2015 \\
\hline F\#13 & 15 de março de 2015 \\
\hline F\#14 & 15 de março de 2015 \\
\hline F\#15 & 16 de janeiro de 2015 \\
\hline F\#16 & 15 de novembro de 2014 \\
\hline F\#17 & 15 de novembro de 2014 \\
\hline F\#18 & 15 de novembro de 2014 \\
\hline F\#19 & 15 de novembro de 2014 \\
\hline F\#20 & 15 de novembro de 2014 \\
\hline &
\end{tabular}

Após a coleta do corpus, cada fotografia foi analisada usando os três significados propostos pela Gramática do Design Visual: significados representativos, significados interacionais e significados composicionais (KRESS, VAN LEEUWEN; 2006). Após a identificação das categorias em cada um dos três significados da GVD, os padrões foram quantificados e interpretados. $\mathrm{Na}$ próxima seção, apresentaremos os resultados encontrados no corpus. 


\section{RESULTADOS}

A Tabela 2 apresenta os resultados encontrados nas Fotografias separados por significado proposto pela GVD:

Tabela 2. Resultados encontrados no corpus separados por tipo de significado.

\begin{tabular}{|c|c|c|c|}
\hline $\begin{array}{l}\text { Número da } \\
\text { fotografia }\end{array}$ & $\begin{array}{c}\text { Significados } \\
\text { Representativos }\end{array}$ & Significados Interativos & $\begin{array}{c}\text { Significados } \\
\text { Composicionais }\end{array}$ \\
\hline $\mathrm{F} \# 1$ & Estrutura conceitual & $\begin{array}{l}\text { Demanda/ Distância } \\
\text { média }\end{array}$ & Ideal/Real \\
\hline $\mathrm{F} \# 2$ & Estrutura conceitual & Oferta/Distância longa & Centro/Margem \\
\hline F\#3 & Estrutura conceitual & Oferta/Distância longa & Centro/Margem \\
\hline $\mathrm{F \# 4}$ & Estrutura conceitual & Oferta/Distância longa & Centro/Margem \\
\hline F\#5 & Estrutura conceitual & Oferta/Distância longa & Centro/Margem \\
\hline F\#6 & Estrutura conceitual & Oferta/Distância longa & Centro/Margem \\
\hline F\#7 & Estrutura conceitual & Oferta/Distância longa & Centro/Margem \\
\hline F\#8 & Estrutura conceitual & $\begin{array}{l}\text { Demanda/Distância } \\
\text { média }\end{array}$ & Ideal/Real \\
\hline F\#9 & Estrutura conceitual & $\begin{array}{l}\text { Demanda/Distância } \\
\text { média }\end{array}$ & Ideal/Real \\
\hline F\#10 & Estrutura conceitual & $\begin{array}{l}\text { Demanda/Distância } \\
\text { média }\end{array}$ & Ideal/Real \\
\hline F\#11 & Estrutura conceitual & $\begin{array}{l}\text { Demanda/Distância } \\
\text { média }\end{array}$ & Ideal/Real \\
\hline F\#12 & Estrutura conceitual & $\begin{array}{l}\text { Demanda/Distância } \\
\text { média }\end{array}$ & Ideal/Real \\
\hline F\#13 & Estrutura conceitual & $\begin{array}{l}\text { Demanda/Distância } \\
\text { média }\end{array}$ & Ideal/Real \\
\hline $\mathrm{F \# 14}$ & Estrutura conceitual & $\begin{array}{l}\text { Demanda/Distância } \\
\text { média }\end{array}$ & Ideal/Real \\
\hline F\#15 & Estrutura conceitual & $\begin{array}{l}\text { Demanda/Distância } \\
\text { média }\end{array}$ & Ideal/Real \\
\hline F\#16 & Estrutura conceitual & $\begin{array}{l}\text { Demanda/Distância } \\
\text { média }\end{array}$ & Ideal/Real \\
\hline F\#17 & Estrutura conceitual & Oferta/Distância longa & Centro/Margem \\
\hline F\#18 & Estrutura conceitual & $\begin{array}{l}\text { Demanda/Distância } \\
\text { média }\end{array}$ & Ideal/Real \\
\hline F\#19 & Estrutura conceitual & $\begin{array}{l}\text { Demanda/Distância } \\
\text { média }\end{array}$ & Ideal/Real \\
\hline F\#20 & Estrutura conceitual & $\begin{array}{l}\text { Demanda/Distância } \\
\text { média }\end{array}$ & Ideal/Real \\
\hline
\end{tabular}

Na próxima seção, discutiremos os resultados apresentados pela Tabela 2.

\section{DISCUSSÃO}

A partir da Tabela 2, podemos enquadrar as fotografias em dois grupos distintos. 0 primeiro grupo é composto por 13 das 20 fotografias (65\%), enquanto que o segundo grupo corresponde às 7 fotografias restantes (35\%). Os resultados serão discutidos a partir de cada um dos significados da GVD. 
Os significados representativos se referem ao que está sendo representado na imagem e se divide em duas estruturas: narrativa e conceitual. As representações narrativas se caracterizam por ações e podem ser mapeadas por vetores que sugerem movimentação (KRESS; VAN LEEUWEN, 2006). Por sua vez, as representações conceituais focalizam no estado das coisas, não havendo sugestão de movimento e sendo possível descrever e/ou classificar os participantes individualmente nesse tipo de estrutura (KRESS; VAN LEEUWEN, 2006). No que diz respeito aos significados representacionais, $100 \%$ do corpus apresentou a estrutura conceitual. Isto pode ser justificado pelo propósito do projeto unbreakable, que é dar voz a vítimas de abuso. Para cumprir esse propósito, a escolha de estruturas conceituais nas fotografias é mais apropriada uma vez que ela pode individualizar os atributos/características portados pelos participantes, destacando as próprias vítimas bem como os dizeres que as vítimas carregam nas fotografias. A Figura 3 ilustra um exemplo de estrutura conceitual:

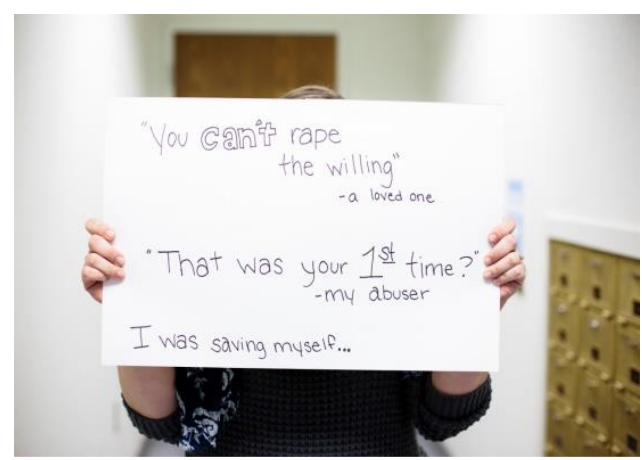

Figura 3 - Exemplo de estrutura conceitual na qual o participante não está identificado. 0 destaque está para o dizer que o participante carrega. (F\#2)

possibilidades: oferta e demanda (KRESS; VAN LEEUWEN, 2006). Uma imagem apresenta uma oferta quando o participante nela representado não mantém contato visual com o leitor e apresenta uma demanda quando o participante mantém contato visual com o leitor justamente para demandar alguma coisa deste (KRESS; VAN LEEUWEN, 2006). No primeiro grupo de fotografias ocorreu a escolha demanda, enquanto que no segundo grupo a escolha oferta pode ser mapeada. Então, pode-se dizer que a maioria das fotografias apresentou demanda porque seus participantes quiseram ser identificados e, além disso, estão chamando a atenção dos leitores de suas fotografias através do contato visual para os abusos sofridos por eles. Já o segundo grupo, apresentou a escolha oferta uma vez que esses participantes preferiram não ser identificados nas fotografias do projeto unbreakable. As Figuras 4 e 5 apresentam exemplos de demanda e oferta encontrados no corpus respectivamente:

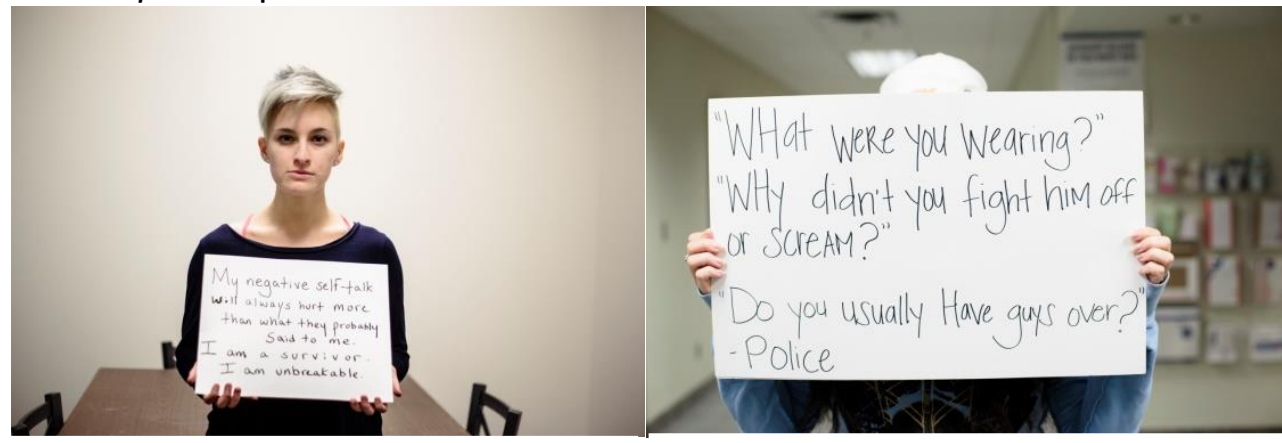

Figura 4 - Exemplo de demanda. 0 Figura 5 - Exemplo de oferta. 0

( participante mantém contato visual $r$ participante não mantém contato rmações na imagem ( com o leitor da imagem. (F\#8) , visual com o leitor da imagem. (F\#3) is principais possibilidades de distribuiçāo de elementos em uma imagem: a) direita/esquerda, onde uma linha vertical pode ser traçada na imagem dividindo-a em direita e esquerda, sendo que a informação à 
esquerda tende a ser mais antiga ou mais familiar aceitável (informação dada) enquanto que a informação à esquerda tende a ser mais nova e menos familiar (informação nova); b) topo/base ou ideal/real, onde uma linha horizontal pode ser traçada na imagem dividindo-a em topo e base, sendo que a informação na base tende a ser mais empírica, concreta e observável enquanto que a informação no topo tende a ser mais abstrata ou algo que pode, deve ou se espera que seja alcançado e c) centro/margem, configuração que posiciona no centro da imagem o que pode ser chamado de informação principal, enquanto que as informações subordinadas ficam às margens (KRESS; VAN LEEUWEN, 2006).

O primeiro grupo de fotografias apresentou a configuração topo/base. A informação que se encontra na base são os dizeres dos abusadores ou de pessoas que souberam do abuso. Isso pode ser justificado uma vez que esses dizeres foram reais e empíricos e pertencentes a um traumatizante acontecimento do passado dessas vítimas. A informação que se encontra no topo é composta pelos rostos das próprias vítimas e pode ser interpretada como a voz por elas alcançada através do projeto unbreakable. Nesse sentido, a configuração topo base torna-se uma escolha apropriada para as vítimas que são identificadas nas fotografias, pois ela proporciona a saída de uma temerosa experiência (real) e caminha para um chamamento dessas pessoas para a sociedade, algo a ser alcançado (ideal). O segundo grupo de fotografias apresentou a configuração centro/margem. Nesse grupo, os participantes das fotografias esconderam seus rostos e, por isso, não são identificados. Dessa forma, os dizeres por eles carregados ficaram em evidência no centro da imagem (informação principal), enquanto que nas margens podemos observar os cenários em que eles se encontram (informações não importantes). As Figuras 6 e 7 ilustram exemplos de configurações topo/base e centro/margem encontrados no corpus respectivamente:

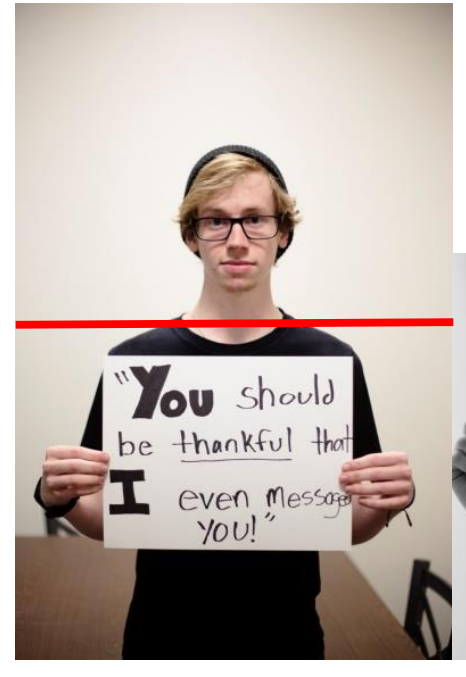

Figura 6 - Exemplo de CONCLUSÃO configuração topo/base. (F\#9)

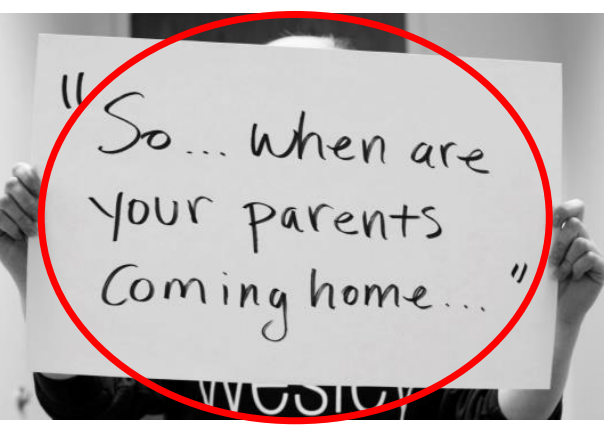

Figura 7 - Exemplo de configuração centro/margem.

(F\#6)

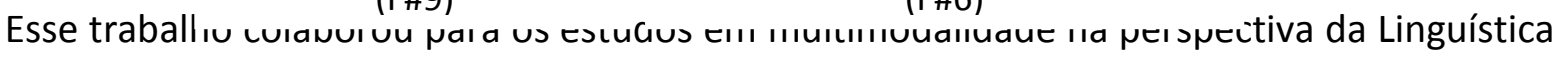
Sistêmico-Funcional, campo ainda novo na área de Letras e com poucos pesquisadores no Brasil. Aqui se pode fazer uma descrição das imagens usando alguns dos sistemas das categorias propostas pela Gramática do Design Visual (KRESS; VAN LEEUWEN, 2006) fundamentada pelo contexto em que essas fotografias foram publicadas, bem como o propósito do projeto unbreakable. Nesse sentido, novos e mais profundos estudos são recomendáveis utilizando-se de um corpus maior e contemplando outros sistemas dentro dos três significados da GVD. Além disso, percebemos a grande importância da linguagem verbal nas fotografias do projeto unbreakable. Por isso, pesquisas sobre as relações intersemióticas entre as linguagens verbal e não verbal são de suma relevância para um melhor entendimento da composição dessas fotografias. 


\section{REFERÊNCIAS}

BEZERRA, F. A. S.; NASCIMENTO, R. G. The role of multimodality labs in multiliteracy projects. I/ha do Desterro, n.64, p. 135-171, 2013.

COPE, B.; KALANTZIS, M. (Eds.). Multiliteracies: literacy learning and the design of social futures. London/New York: Routledge, 2000.

HALLIDAY, M. A. K. An introduction to functional grammar. 3. ed. Revisado por Christian M.I.M. Matthiessen. London: Arnold, 2004.

An introduction to functional grammar. 4. ed.. Revised by Christian M.I.M. Matthiessen. London: Arnold, 2014.

KRESS, G.; van LEEUWEN, T. Reading images: the grammar of visual design. 2 ed. London: Routledge, 2006.

PROJETO UNBREAKABLE. Project Unbreabkable. Disponível em <http://projectunbreakable.tumblr.com/> ; acessado em 2 de maio de 2016. 